\title{
Alta dirección pública y techo de cristal.Acceso de las mujeres a los puestos directivos en Chile
}

\section{Public senior management and glass ceiling. Access of women to management positions at Chile}

\author{
doi: https://doi.org// 0.32870/ \\ eees.v27i77.7085
}

\section{Resumen}

Este artículo presenta los resultados de una investigación cuyo objetivo fue analizar el fenómeno del techo de cristal (TC) en el sector público chileno, a partir de la representación de mujeres en los nombramientos de los concursos de alta dirección pública desarrollados entre 2010 y 2017. Se utilizaron procedimientos de estadística descriptiva para examinar una base de datos cuantitativos obtenidos del Servicio Civil en Chile. Se observa que solamente el $29 \%$ de los concursos realizados fueron adjudicados a mujeres. Se concluye que existe una importante segregación vertical en los nombramientos de los concursos de primer y segundo nivel jerárquico de la administración del Estado en Chile, lo que permite inferir la existencia de una desigualdad de género que configura el TC en dicho sistema.

Palabras clave: discriminación de género, segregación vertical, administración pública, techo de cristal, Chile.

\author{
Ricardo Gaete Quezada \\ Juliana Álvarez Rodríguez*•
}

\begin{abstract}
This article presents the results of a research which objective is to analyze the phenomenon of the glass ceiling in the Chilean public sector, from the representation of women in the appointments of the Senior Public Management competitions developed between 2010 and 2017. Descriptive statistics procedures were used to examine a quantitative database obtained from the Civil Service in Chile, noting that only $29 \%$ of the contests were adjudicated to women, concluding that there is an important vertical segregation in the appointments of the first competitions and second hierarchical level of the state administration in Chile, which allows to infer the existence of gender inequality in said system that configures the glass ceiling.
\end{abstract}

Keywords: Gender discrimination, vertical segregation, public administration, glass ceiling, Chile.

\footnotetext{
-Profesor-Investigador de la Universidad de Antofagasta, Chile. ORCID: https://orcid.org/00000003-2359-2304_ricardo.gaete@uantof.cl

- Profesora-Investigadora de la Universidad Juárez Autónoma de Tabasco, México. ORCID: https://orcid.org/0000-000 I-683 I-4303_juliana.alvarez@ujat.mx

Fecha de recepción: 21 de enero de 2019. Fecha de aceptación: II de agosto de 2019.
} 
Introducción

El Sistema de Alta Dirección Pública de Chile (SADP) fue instaurado en 2003 a través de la Ley 19.882 (González, Olivares, Abarca, y Molina, 2016; Pliscoff, 2016; Egaña, 2015; Llano, 2014), y se transformó en un hito de modernización del Estado chileno sustentado en una serie de pilares fundamentales relacionados con la idoneidad y el mérito, la transparencia y la igualdad de oportunidades entre los candidatos.

El sistema fue desarrollado como respuesta a la crisis política que afectaba al país en aquella época (Fraile, 2018; González et al., 2016; Pliscoff, 2016; Williamson e Irarrázabal, 2016; Maillet, 2015), debida al abuso en la discrecionalidad con que se otorgaban los nombramientos para los cargos directivos del Gobierno central, mayormente basados en la confianza política y no en la capacidad profesional. Esto detonaría una serie de modificaciones mediante la Ley 20.955 del 2016 (Williamson e Irarrázabal, 2016) para perfeccionar el sistema y fortalecer a la Dirección Nacional del Servicio Civil (DNSC).

El presente artículo analiza la problemática del techo de cristal (TC) en los concursos de alta dirección pública (ADP) realizados en Chile durante un periodo de ocho años, que abarca el primer Gobierno del presidente Sebastián Piñera Echeñique (2010-2013) y el segundo de la presidenta Michelle Bachelet Jeria (2014-2017).

Los datos se analizan mediante procedimientos de estadística descriptiva, y se observa que el 29\% de los concursos de ADP realizados durante el periodo bajo estudio fue adjudicado a mujeres, además de que el Ministerio de Desarrollo Social (52\%) fue la institución en donde se alcanzó un mayor porcentaje de concursos así adjudicados, y de que en el Ministerio de Minería (7\%) se registró el porcentaje más bajo.

A partir de los resultados obtenidos, es posible concluir que existe una importante segregación vertical en el SADP 
que configura un Tc, y que se expresa en una representación desequilibrada entre hombres y mujeres en los nombramientos de los concursos de ADP en Chile, lo que coincide con la actual distribución de cargos de exclusiva confianza de la administración pública centralizada chilena.

\section{Revisión de la literatura}

\section{I.I Techo de cristal en el sector público}

$\mathrm{El}$ TC es la metáfora dominante en las ciencias sociales desde finales de la década de los ochenta para describir los desafíos que enfrentan las mujeres para acceder a puestos de mayor responsabilidad en sus organizaciones laborales (Carrancio, 2018; Caceres-Rodriguez, 2013; Sabharwal, 2013; Cuadrado y Morales, 2007). Dicha problemática se relaciona con barreras invisibles, tales como la falta de capital humano, estereotipos basados en el género, diferencias en los estilos de comunicación, exclusión de redes informales, limitado apoyo a programas de conciliación trabajo-familia, falta de mentores y modelos a seguir, segregación laboral por sexo, y sesgos actitudinales y organizativos (Sabharwal, 2013, p. 400).

De acuerdo con los estudios de género, la diferencia de roles que tanto mujeres como hombres desempeñan dentro de la sociedad no son hechos naturales o biológicos, por el contrario, se trata de constructos culturales. Así, Gutiérrez (2016, p. 359) afirma que "la desigualdad entre ambos [géneros] surge debido a preceptos y valores que las sociedades tienen preconcebidos, no fortuitamente, sino [provocados] por el predominio del patriarcado", lo que permite explicar en gran medida las principales raíces del TC para las mujeres en los ámbitos laboral, académico, político, etc.

De esta manera, Macarie y Moldovan (2014, p. 10) afirman que el TC en esencia "es un fenómeno de discriminación vertical de género que se refiere al hecho de que las mujeres 
usualmente están subrepresentadas en cargos gerenciales superiores", lo que plantea una problemática social que discrimina a las mujeres respecto de los hombres en el desarrollo de sus carreras laborales.

Diferentes instancias internacionales, como el Instituto de Investigación de las Naciones Unidas para el Desarrollo Social (UNRISD, por sus siglas en inglés) (2006), la Comisión Económica para América Latina y el Caribe (CEPAL) (2014) o la Organización Internacional del Trabajo (OIT) (2017), han realizado diferentes estudios que coinciden en identificar un relativo avance en el logro de la meta del 30\%, acordada por los Estados miembros de Naciones Unidas en Beijing en 1995, en el acceso de las mujeres a cargos directivos en las últimas dos décadas.

Según Gutiérrez (2016), durante la cuarta conferencia mundial sobre mujeres de Naciones Unidas, se estableció el principio de transversalidad de género (gender mainstreaming), que entre otros aspectos señala que "las mujeres deben incorporarse a aquellos espacios de poder y al ámbito del trabajo remunerado[,] y los hombres deben participar en el ámbito del trabajo doméstico y de cuidado de las personas dependientes" (p. 364), lo que otorgó el contexto adecuado para que los Estados implementaran las acciones necesarias para avanzar hacia la meta del tercio de mujeres en cargos de dirección superior.

Respecto al TC en la administración pública a nivel mundial, el Programa de las Naciones Unidas para el Desarrollo (PNUD) (2014) señala, con respecto al objetivo mínimo del $30 \%$ de mujeres en puestos directivos acordada en Beijing, que "si bien se ha avanzado en términos de cantidades totales de mujeres en la administración pública, techos y muros de cristal siguen presentando desafíos en cuanto a la participación igualitaria de las mujeres en los puestos de toma de decisiones" (p. 8), lo que denota que la carrera 
laboral de las mujeres debe enfrentar un sinnúmero de obstáculos por discriminaciones basada en el género.

En la Tabla 1, se visualiza la existencia de diferentes estudios e investigaciones sobre el TC en el sector público a nivel mundial, desarrollados en los últimos años. Los estudios incluidos son una muestra que confirma la tendencia observada globalmente en diferentes sectores económicos (Programa de las Naciones Unidas para el Desarrollo, 2014), según la cual las mujeres han aumentado en cuanto a su número total de participación en el sector laboral público (Carrancio, 2018; Comisión Nacional de Derechos Humanos de México, 2017), aunque dicho aumento no se ha reflejado en la misma medida cuando se analizan específicamente los cargos directivos en las administraciones públicas, donde las mujeres ocupan entre 30 y 40\% de puestos (Carrancio, 2018; Corte Suprema de Justicia de Argentina, 2014; Macarie y Moldovan, 2014). 


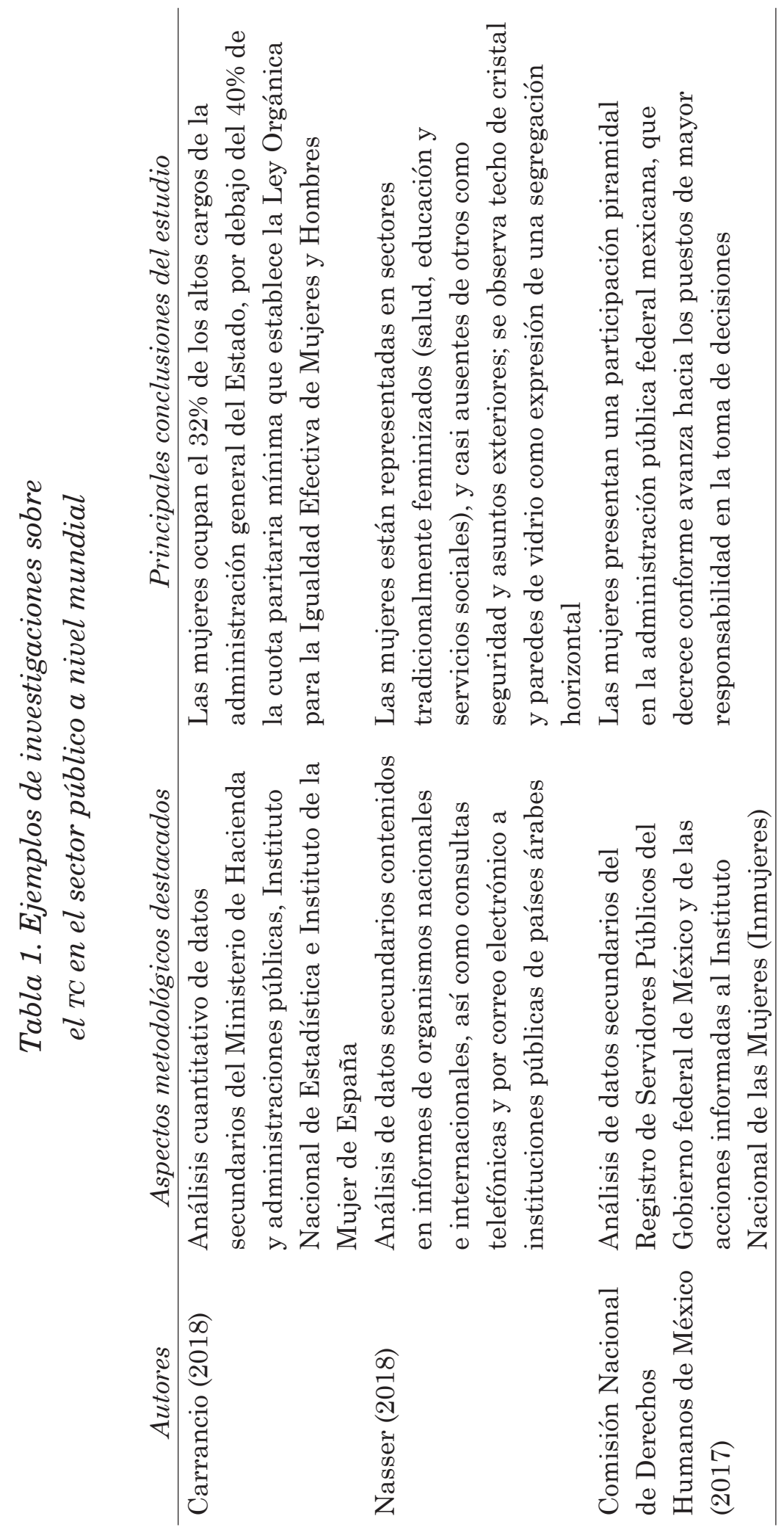




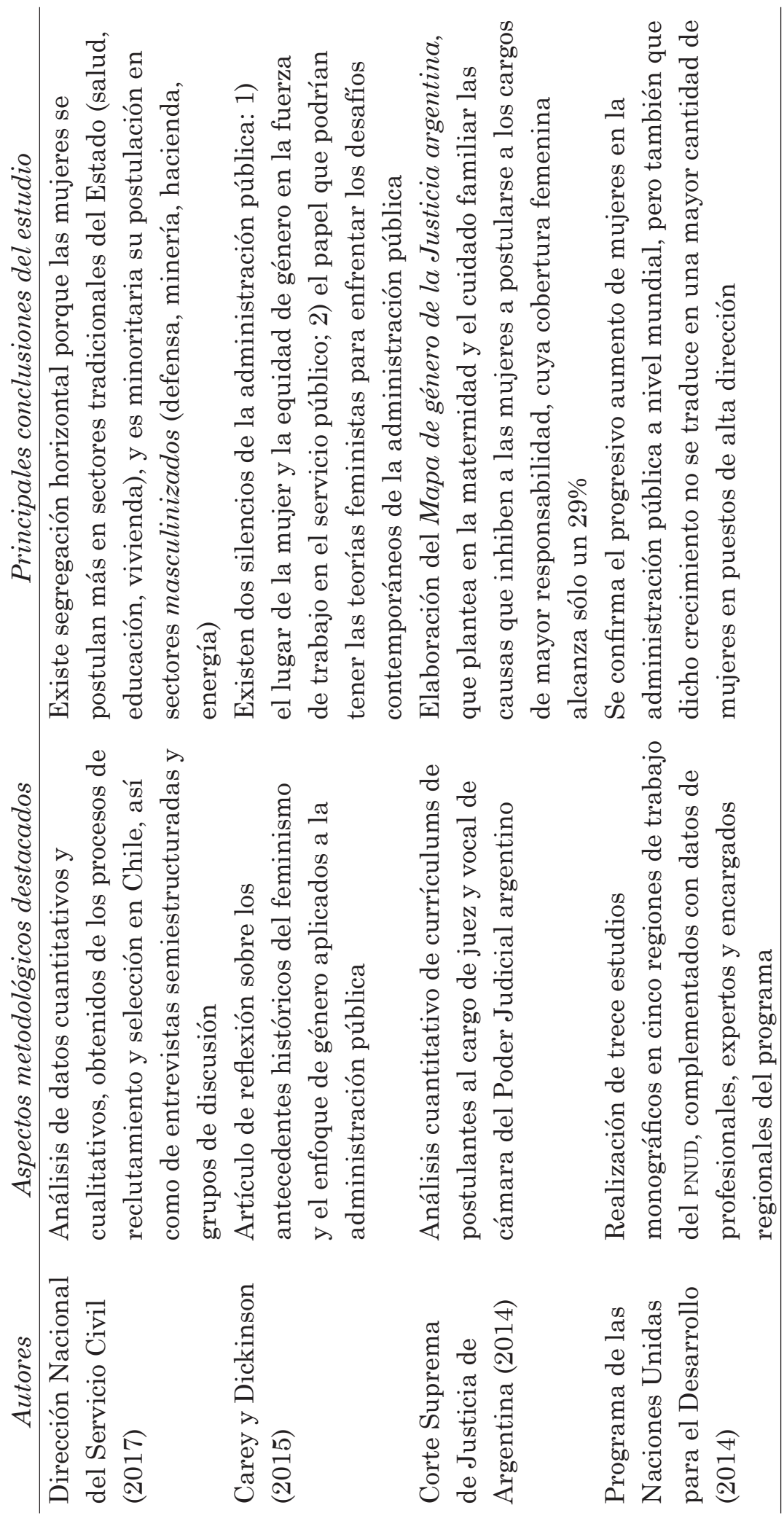

Sociedad No.77 
Ricardo Gaete Quezada y Juliana Álvarez Rodríguez

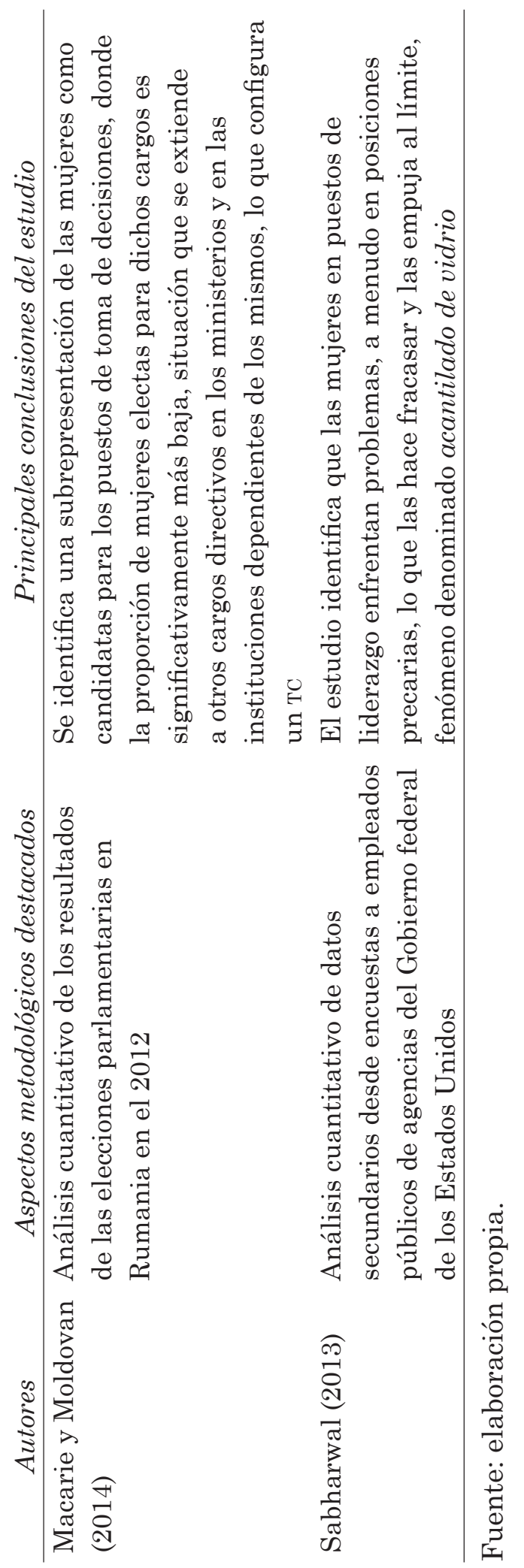

\section{6}


La mayoría de las investigaciones sobre el TC en el sector público incluidas en la Tabla 1 fueron desarrolladas con un enfoque cuantitativo, mediante la revisión de datos obtenidos desde fuentes secundarias, con la excepción del estudio realizado por la Dirección Nacional del Servicio Civil (2017) para el caso chileno, que combina el análisis de datos cuantitativos con la aplicación de técnicas de recolección de información de tipo cualitativa (entrevistas y grupos de discusión).

Los estudios expuestos en la Tabla 1 confirman tres grandes hallazgos de interés para el estudio del Tc, algunos ya mencionados anteriormente: 1) la participación de las mujeres en cargos directivos de mayor responsabilidad a nivel estatal se aproxima a la meta establecida en Beijing en 1995; 2) existe una segregación vertical en cargos directivos en sectores tradicionalmente masculinos en las administraciones públicas, no obstante el creciente aumento de mujeres trabajando en los diferentes ámbitos del sector público; y 3) también se constata una segregación horizontal, que encasilla a las mujeres en sectores laborales específicos como salud o educación, y las relega de otros ámbitos en donde la cantidad de hombres es históricamente mayoritaria.

En virtud de lo anterior, según el Programa de las Naciones Unidas para el Desarrollo (2014), es posible afirmar que

En muchos contextos nacionales donde la cantidad de mujeres iguala a la de hombres en la administración pública como un todo, las mujeres tienden a agruparse en puestos de menor rango, con menos prestigio y sueldos más bajos[,] y en aquellos que tradicionalmente se consideran sectores "ligeros" o feminizados, como cultura, educación, salud y turismo. En la mayoría de los países[,] las mujeres están infrarrepresentadas, si es que están presentes, en sectores como seguridad, finanzas y planificación [,] que siguen siendo dominados por hombres. (p. I2)

El planteamiento del Programa de las Naciones Unidas para el Desarrollo (2014) no sólo identifica una segregación verti- 
cal relacionada con el TC en el Estado, sino que además deja ver que en las administraciones públicas a nivel mundial también existe una segregación horizontal, relacionada con el concepto de muros de cristal (Nasser, 2018; Moldovan, 2016; Cárdenas, Correa, y Prado, 2014; Sabharwal, 2013), que plantea que las mujeres no pueden acceder de manera igualitaria que los hombres a determinados sectores laborales dentro del Estado, a diferencia del Tc, que alude a una segregación de tipo vertical (Macarie y Moldovan, 2014).

A pesar de lo planteado en la Tabla 1, Carrancio (2018) afirma que la existencia del TC es más factible en el sector privado que en el público debido a que en este último deberían desarrollarse procesos de carrera laboral igualitarios entre hombres y mujeres:

La dificultad de acceso a los puestos más altos de la escala jerárquica parece ser una práctica más común en la empresa privada, puesto que la administración pública, en su legislación, se muestra imparcial y transparente tanto en la selección como en la promoción de su personal, basándose en los principios constitucionales de igualdad, mérito y capacidad que garantizan la igualdad de oportunidades en el empleo. (p. 476)

Sin embargo, la mirada legalista de la administración pública expuesta anteriormente no es garantía suficiente para evitar la discriminación laboral hacia las mujeres relacionada con el TC en el sector público en la actualidad a nivel mundial, especialmente en cuanto al acceso de las mujeres a los cargos directivos de mayor responsabilidad en la toma de decisiones.

Además, las mujeres que ocupan posiciones de liderazgo igualmente deben enfrentar situaciones precarias, que las empujan a dejar ese tipo de puestos directivos en las instituciones estatales (Sabharwal, 2013), lo que a su vez se relaciona con otras formas de expresión de la discriminación hacia las mujeres como la doble carga laboral o menores 
remuneraciones por funciones similares a las que desempeñan los hombres.

I.2 Segregación laboral vertical en el ámbito estatal chileno Para el caso de Chile, según Carrera (2016), la discriminación por razones de género en la administración pública del país es un tema pendiente, especialmente en lo relativo a los cargos directivos en donde las mujeres se encuentran subrepresentadas, en las instituciones tanto centralizadas como descentralizadas. En la Figura 1, se exponen las principales características de la estructura de la administración pública chilena, regida por la Ley 18.575 Orgánica Constitucional de Bases Generales de la Administración del Estado.

Figura 1. Estructura de la administración pública chilena, según Ley 18.575

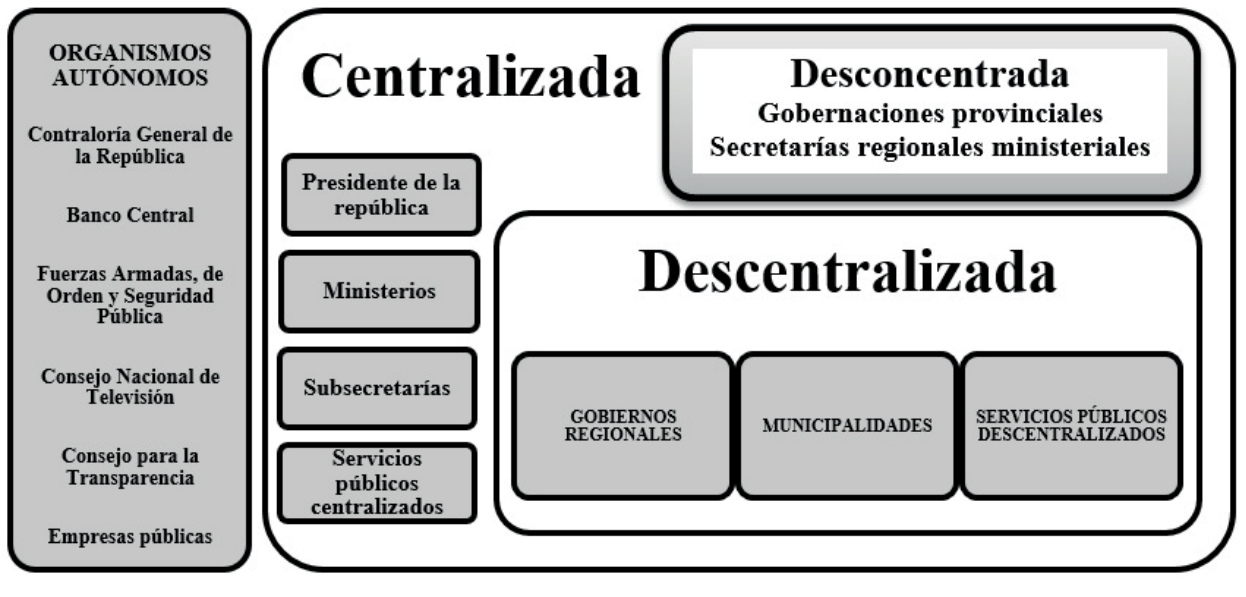

Fuente: elaboración propia.

La Ley 18.575 establece que los presidentes de la república tienen la potestad de nombrar a funcionarios de su exclusiva confianza en los veinticuatro ministerios y las treinta y siete subsecretarias, así como en las dieciséis intenden- 
cias regionales y en las cincuenta y seis gobernaciones provinciales. En la Tabla 2, según datos de la Dirección de Presupuestos (DIPRES) (2019), se presenta la distribución de los funcionarios públicos según su género en los ministerios del Gobierno central, a marzo de 2019.

Tabla 2. Distribución por género del personal en los ministerios del Gobierno central de Chile (a marzo de 2019)

\begin{tabular}{ccc} 
Ministerios & Hombres & Mujeres \\
\hline Interior & $46.0 \%$ & $54.0 \%$ \\
Relaciones Exteriores & $52.5 \%$ & $47.5 \%$ \\
Economía & $45.7 \%$ & $54.3 \%$ \\
Hacienda & $51.1 \%$ & $48.9 \%$ \\
Educación & $21.1 \%$ & $78.9 \%$ \\
Justicia & $61.7 \%$ & $38.3 \%$ \\
Defensa & $68.4 \%$ & $31.6 \%$ \\
Obras Públicas & $65.9 \%$ & $34.1 \%$ \\
Agricultura & $56.9 \%$ & $43.1 \%$ \\
Bienes Nacionales & $46.6 \%$ & $53.4 \%$ \\
Trabajo & $44.2 \%$ & $55.8 \%$ \\
Salud & $33.0 \%$ & $67.0 \%$ \\
Minería & $62.4 \%$ & $37.6 \%$ \\
Vivienda & $47.4 \%$ & $52.6 \%$ \\
Transportes y Telecomunicaciones & $60.0 \%$ & $40.0 \%$ \\
Secretaría General de Gobierno & $50.2 \%$ & $49.8 \%$ \\
Desarrollo Social & $41.6 \%$ & $58.4 \%$ \\
Secretaría General de la Presidencia & $54.7 \%$ & $45.3 \%$ \\
Energía & $58.7 \%$ & $41.3 \%$ \\
Medio Ambiente & $44.6 \%$ & $55.4 \%$ \\
Del Deporte & $60.5 \%$ & $39.5 \%$ \\
De la Mujer & $26.5 \%$ & $73.5 \%$ \\
De las Culturas & $46.4 \%$ & $53.6 \%$ \\
\hline
\end{tabular}

Fuente: Dirección de Presupuestos (2019, p. 30).

Con base en lo planteado en la Tabla 2, los ministerios de Educación, Salud y de la Mujer son los que registran una

\section{0}


alta participación de mujeres en dichas instituciones, por sobre el 70\% respecto de los hombres. Por contrapartida, los ministerios de Defensa, Minería y Obras Públicas son los que registran una alta participación de hombres, por sobre el $60 \%$ respecto de las mujeres.

En la Tabla 3, se presenta la distribución por género a junio de 2019 del personal de cargos directivos en ministerios y subsecretarías del país. Ministros y subsecretarios pertenecen al espectro de nombramientos de funcionarios de exclusiva confianza del presidente de la república de Chile en la administración centralizada, según la estructura expuesta anteriormente en la Figura 1, por ende, son los encargados de la toma de decisiones de las políticas públicas desarrolladas en el país.

Tabla 3. Distribución por género de cargos directivos en ministerios y subsecretarías en Chile (Gobierno de Sebastián Piñera, a junio de 2019)

\begin{tabular}{ccccc} 
& \multicolumn{2}{c}{ Ministros/as } & \multicolumn{2}{c}{ Subsecretarios/as } \\
Ministerio & Hombre & Mujer & Hombre & Mujer \\
\hline Salud & 1 & 0 & 1 & 1 \\
Energía & 1 & 0 & 1 & 0 \\
Agricultura & 1 & 0 & 1 & 0 \\
Trabajo y Previsión Social & 1 & 0 & 1 & 1 \\
Del Deporte & 0 & 1 & 1 & 0 \\
Justicia y Derechos Humanos & 1 & 0 & 1 & 1 \\
Interior y Seguridad Pública & 1 & 0 & 2 & 1 \\
Educación & 0 & 1 & 1 & 1 \\
Hacienda & 1 & 0 & 1 & 0 \\
Desarrollo Social y Familia & 1 & 0 & 1 & 2 \\
Defensa Nacional & 1 & 0 & 2 & 0 \\
Economía, Fomento y Turismo & 1 & 0 & 2 & 1 \\
Medio Ambiente & 0 & 1 & 1 & 0 \\
Relaciones Exteriores & 1 & 0 & 0 & 1 \\
Obras Públicas & 1 & 0 & 1 & 0 \\
Minería & 1 & 0 & 1 & 0 \\
\hline
\end{tabular}

Sociedad No.77 
Ricardo Gaete Quezada y Juliana Álvarez Rodríguez

\begin{tabular}{ccccc} 
& \multicolumn{2}{c}{ Ministros/as } & \multicolumn{2}{c}{ Subsecretarios/as } \\
Ministerio & Hombre & Mujer & Hombre & Mujer \\
\hline De la Mujer y la Equidad de & 0 & 1 & 0 & 1 \\
Género & & & & \\
Vivienda y Urbanismo & 1 & 0 & 1 & 0 \\
$\quad$ Transportes y & 0 & 1 & 1 & 1 \\
Telecomunicaciones & & & & \\
Bienes Nacionales & 1 & 0 & 0 & 1 \\
Cultura y las Artes & 0 & 1 & 2 & 0 \\
Ciencia, Tecnología, & 1 & 0 & 0 & 1 \\
Conocimiento e Innovación & & & & \\
Secretaría General de Gobierno & 0 & 1 & 1 & 0 \\
Secretaría General de la & 1 & 0 & 1 & 0 \\
Presidencia & & & & \\
Total & 17 & 7 & 24 & 13 \\
\hline
\end{tabular}

Fuente: elaboración propia.

En la Tabla 3, se observa que la distribución por género del cargo de ministro muestra que actualmente sólo el 29\% es desempeñado por mujeres, mientras que en el caso del cargo de subsecretario el porcentaje es de $35 \%$, resultados similares a los obtenidos en otras investigaciones sobre las administraciones públicas de países como España (Carrancio, 2018) o Rumania (Moldovan, 2016).

Además, según la Tabla 3, un total de nueve ministerios (Agricultura, Energía, Hacienda, Defensa Nacional, Obras Públicas, Minería, Vivienda y Urbanismo, Bienes Nacionales y Secretaría General de la Presidencia) son dirigidos completamente por hombres, dado que tanto el cargo de ministro como el de subsecretario son desempeñados por un sujeto de dicho género, lo que expresa una segregación horizontal en la administración centralizada del Estado chileno, con sectores donde las mujeres no están representadas.

Por el contrario, de acuerdo también con la Tabla 3, únicamente el Ministerio de la Mujer y la Equidad de Género cuenta con mujeres tanto en los cargos de ministra como de subsecretaria, por lo que el caso chileno muestra una subre- 
presentación de las mujeres incluso en aquellos sectores de la administración pública tradicionalmente feminizados, como salud, educación o servicios sociales (Nasser, 2018).

Asimismo, según la Dirección de Presupuestos (2018, p. 17), en la administración pública centralizada chilena existen tres clases de puestos en el estamento directivo del personal civil:

- Autoridades de Gobierno y jefes superiores de servicio. Considera a autoridades de Gobierno tales como ministros y subsecretarios, presidente de la Corte Suprema y los jefes superiores de los servicios e instituciones de la cobertura.

- Directivos profesionales. Considera al personal que se desempeña en un cargo directivo y que percibe asignación profesional; incluye también al personal en cargos directivos de servicios fiscalizadores y al personal del escalafón superior de las instituciones del Poder Judicial.

- Directivos no profesionales. Considera a personal que se desempeña como directivo que no percibe asignación profesional y al personal de servicios fiscalizadores que desempeña un cargo de jefatura.

Tabla 4. Personal civil del estamento directivo del Gobierno central de Chile, según sexo (cantidades y porcentajes)

\begin{tabular}{lccccccccc} 
& & 2010 & 2011 & 2012 & 2013 & 2014 & 2015 & 2016 & 2017 \\
\hline $\begin{array}{l}\text { Autoridades } \\
\text { de Gobierno }\end{array}$ & $\mathrm{H}$ & 164 & 172 & 178 & 180 & 182 & 170 & 182 & 167 \\
\cline { 2 - 9 } $\begin{array}{l}\text { y jefes } \\
\begin{array}{l}\text { superiores de } \\
\text { servicio }\end{array}\end{array}$ & $\mathrm{M}$ & $\begin{array}{c}69 \\
30 \%\end{array}$ & $\begin{array}{c}60 \\
26 \%\end{array}$ & $\begin{array}{c}63 \\
26 \%\end{array}$ & $\begin{array}{c}67 \% \\
27 \%\end{array}$ & $\begin{array}{c}66 \\
27 \%\end{array}$ & $\begin{array}{c}68 \\
29 \%\end{array}$ & $\begin{array}{c}71 \\
20 \%\end{array}$ & $\begin{array}{c}83 \\
3\end{array}$ \\
\hline $\begin{array}{l}\text { Directivos } \\
\text { profesionales }\end{array}$ & $\mathrm{H}$ & 3950 & 3690 & 3822 & 3881 & 4003 & 3867 & 3950 & 4040 \\
\cline { 2 - 9 } & $\mathrm{M}$ & $\begin{array}{c}2820 \\
42 \%\end{array}$ & $42 \%$ & $42 \%$ & $42 \%$ & $42 \%$ & $42 \%$ & $42 \%$ & $42 \%$ \\
\hline
\end{tabular}

Sociedad No.77 
Ricardo Gaete Quezada y Juliana Álvarez Rodríguez

\begin{tabular}{lccccccccc} 
& & 2010 & 2011 & 2012 & 2013 & 2014 & 2015 & 2016 & 2017 \\
\hline Directivos no & $\mathrm{H}$ & 869 & 820 & 739 & 782 & 823 & 818 & 791 & 702 \\
\cline { 2 - 9 } profesionales & & 451 & 389 & 360 & 350 & 364 & 329 & 337 & 301 \\
& $\mathrm{M}$ & $34 \%$ & $32 \%$ & $33 \%$ & $31 \%$ & $31 \%$ & $29 \%$ & $30 \%$ & $30 \%$ \\
\hline
\end{tabular}

Fuente: elaboración propia con base en Dirección de Presupuestos (2018).

La Tabla 4 muestra una menor presencia de las mujeres en los cargos del estamento directivo del personal civil de la administración centralizada, especialmente para los casos de las autoridades de Gobierno y los directivos no profesionales, que tienen un $30 \%$ de mujeres. Lo anterior coincide con lo señalado en la Tabla 3 para el caso del Gobierno del presidente Sebastián Piñera, al menos hasta junio de 2019.

Finalmente, un antecedente importante respecto de la participación de las mujeres en cargos públicos chilenos ocurrió durante el primer Gobierno de la presidenta Michelle Bachelet Jeria, que realizó la publicación del Código de buenas prácticas laborales (CBPL) sobre no discriminación. En la Tabla 5, se resume el objetivo del código, relacionado con la representación equilibrada o paritaria entre hombres y mujeres en los cargos de jefatura y responsabilidad directiva en la administración central del Estado chileno. 
Alta dirección pública y techo de cristal. Acceso de las mujeres a los puestos directivos en Chile

Tabla 5. Representación equilibrada entre hombres y mujeres en cargos de jefatura y de responsabilidad directiva según objetivos del código

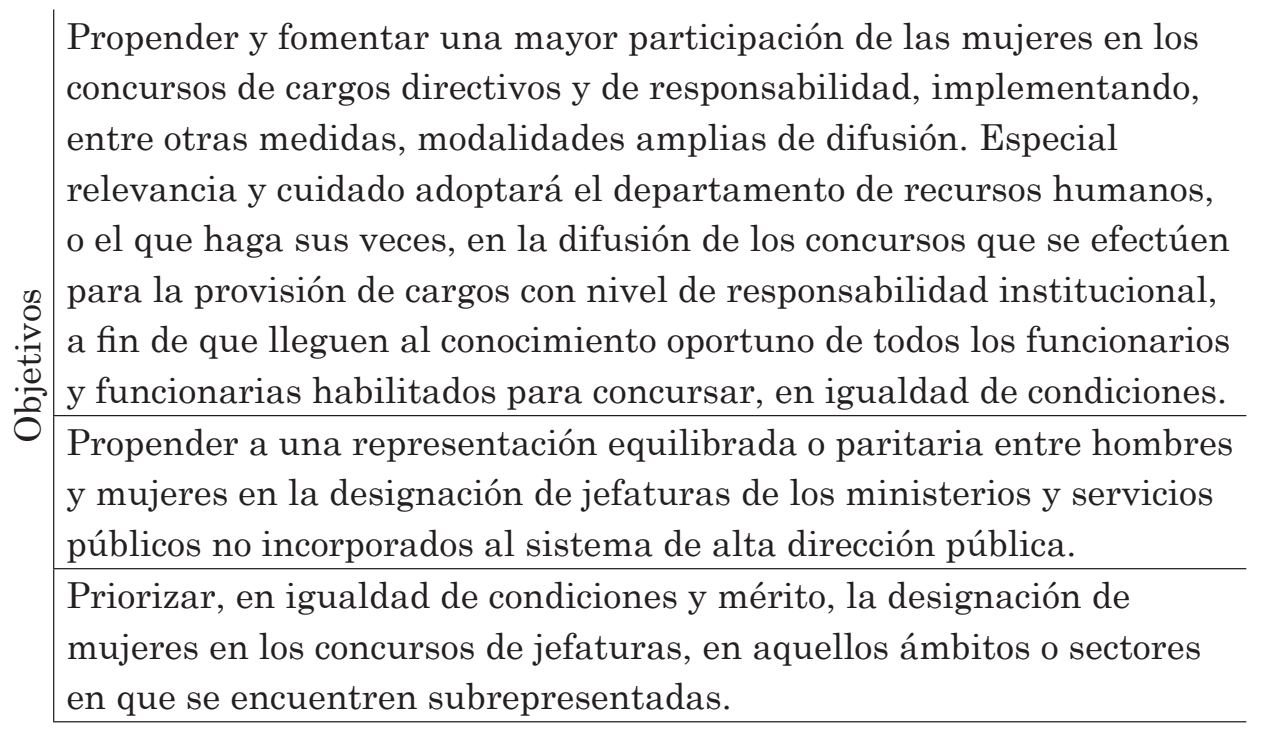

Fuente: adaptación de Servicio Nacional de la Mujer (2008, pp. 15-16).

Respecto de la Tabla 5, resulta especialmente interesante para el presente estudio el planteamiento del CBPL de priorizar a las mujeres en el desempeño de un cargo directivo como una acción afirmativa para obtener una representación más equilibrada entre hombres y mujeres en sectores en donde estas últimas se encuentren subrepresentadas, en concordancia con lo estipulado por el artículo 7 de la Convención sobre la eliminación de todas las formas de discriminación contra la mujer, elaborada en 1979 por el Comité para la Eliminación de la Discriminación contra la Mujer (CEDAw, por sus siglas en inglés) (1979).

De esta manera, a partir de los antecedentes expuestos, se observa que el caso del Gobierno chileno evidencia una importante segregación vertical y horizontal hacia las mujeres y, estadísticamente, la configuración de un TC respecto de los cargos directivos de exclusiva confianza del presidente de la república de Chile en la administración

Sociedad No.77 
pública centralizada, por lo que cabe preguntarse si dicha situación se manifiesta de igual manera en el SADP en Chile.

\section{Sistema de Alta Dirección Pública en Chile}

El SADP fue instaurado en Chile a través de la Ley 19.882 del Ministerio de Hacienda, promulgada el 11 de junio de 2003, que establece una nueva política de personal para los funcionarios públicos chilenos que se transformó en una experiencia pionera de modernización del Estado en América Latina (Iacoviello, Llano, y Ramos, 2017; Ortega, 2016; Zuvanic, 2016; Egaña, 2015; Longo, 2013), y que considera los principales aspectos del modelo norteamericano (Fraile, 2018).

Según Pliscoff (2016), el origen de la ADP se encuentra relacionado con el paradigma de la nueva gestión pública (NGP), que en la década de los noventa renovó el tradicional sistema burocrático en la administración, y que establece como uno de sus aspectos característicos el estatus especial que le asigna a los niveles gerenciales y a la figura del gerente público.

Ahora bien, la génesis de este hito de la modernización del Estado chileno que fue el SADP se encuentra relacionada con una crisis política generada por la preeminencia de la confianza política en el nombramiento discrecional de los directivos públicos adscritos al Gobierno central, esto por sobre la idoneidad y el mérito técnico que el candidato tuviera para ejercer el puesto directivo (Fraile, 2018; Servicio Civil, 2018; Espinoza, 2017; Ortega, 2016; Pliscoff, 2016; Zuvanic, 2016; Williamson e Irarrázabal, 2016; Maillet, 2015; Llano, 2014; Longo, 2013). Según González et al. (2016), se trataba de sistemas de botín, un clientelismo que afectaba las designaciones al priorizar la confianza política sobre las competencias técnicas.

\section{6}


Al cumplirse quince años del funcionamiento del SADP, se ha registrado un paulatino aumento en el número de instituciones estatales y cargos directivos incorporados desde su creación (Espinoza, 2017; Llano, 2014).

Ahora bien, es especialmente relevante la crisis de legitimidad del SADP ocurrida en los últimos tres cambios de Gobierno nacional, que han estado caracterizados por la alternancia de las coaliciones de gobierno, lo que según Ortega (2016, p. 2) ha implicado que "se están seleccionando altos directivos dentro del circulo de los funcionarios de la misma coalición en los distintos Gobiernos de turno, lo contrario a una selección amplia y apolítica independiente de la coalición gobernante", y que es también contrario a los principios fundamentales que dieron origen al sistema.

Asimismo, Maillet (2015, p. 16) señala que en el caso del SADP chileno "se trata de un modelo híbrido que balancea profesionalización y politización de los cargos de la alta función pública", lo que es uno de los aspectos más sensibles de la experiencia chilena, pues para alcanzar dicho equilibrio se han desarrollado procesos de negociación entre la autoridad política y los altos directivos públicos, materializados en la firma de convenios de desempeño, lo que a su vez, según Pliscoff (2016, p. 398) genera un "problema de coordinación intersectorial" que fragmenta el accionar del Estado frente a los problemas que están incorporados en la agenda pública.

Según el Servicio Civil (2018), el periodo 2003-2017 ha permitido recorrer un camino hacia la profesionalización de la alta dirección pública en Chile, caracterizada entre otras cuestiones por:

- Profesionalización de los directivos públicos: criterios meritocráticos para seleccionar líderes profesionales en instituciones estatales que ejecutan políticas públicas.

Sociedad No.77 
- Régimen diferenciado: enfocado exclusivamente en los cargos de primer y segundo nivel jerárquico subordinados al poder político.

- Sistema mixto político-meritocrático: introduce procedimientos científicos para equilibrar las consideraciones técnicas y políticas inherentes al directivo público.

- Institucionalidad gestora garante y especializada: el Consejo de Alta Dirección Pública es la entidad responsable de velar por el correcto funcionamiento del sistema.

- Especificidad de cargos y apertura a la sociedad: oferta de cargos directivos públicos con un perfil del puesto específico y abierta para profesionales del sector público y privado.

- Reclutamiento y selección externos y transparentes: participación de diferentes instancias institucionales, así como consultoras especializadas en selección de directivos.

- Enfoque contractualista del desempeño directivo: los convenios de desempeño fijan las prioridades, objetivos e incentivos de gestión para los directivos públicos chilenos.

- Equilibrio en el gerenciamiento del sistema: el proceso de selección está a cargo del Servicio Civil, y la autoridad política es responsable desde la firma del convenio.

Sin embargo, Zuvanic (2016) identifica como una de las falencias del SADP a la fuerte discrecionalidad de los presidentes de la república, quienes pueden elegir por criterios de confianza política entre los finalistas de las ternas en cada concurso de ADP, pedirles la renuncia en cualquier momento por cualquier razón y designar de manera provisoria a directivos públicos suplentes.

Asimismo, Maillet (2015) identifica a la discrecionalidad política como una de las problemáticas clásicas en el análisis 
de la ADP cuando se otorga mayor importancia a la confianza política que a la meritocracia: "en vez de oponer ambos criterios, se tiene especial interés en la coexistencia entre la preocupación por la adecuada calidad profesional de los individuos, y cierta responsabilidad y compromiso político por parte de quienes ocupan los cargos" (p. 12).

A pesar de la ya mencionada gran capacidad discrecional de los presidentes de la república de Chile, esta no se ha traducido en una aplicación de criterios de equidad de género que discrecionalmente favorezcan a las mujeres, de modo que se logre equilibrar su representación con la de los hombres en los puestos directivos en el sector público, lo que según González et al. (2016, p. 72) es fuertemente criticable porque dichos cargos "son de confianza directa del presidente de la república, [por lo que] este antecedente es una mala señal respecto a los avances en igualdad de género que se han promovido en Chile durante la última década”. Lo dicho confirma la apreciación de que la discrecionalidad presidencial debería utilizarse como una acción afirmativa.

De acuerdo con Comunidad Mujer (2015), a nivel empresarial existen tres mecanismos para aumentar la participación de las mujeres, que podrían considerarse como estrategias para equilibrar la presencia de hombres y mujeres en cargos directivos en el sector público chileno:

- Autorregulación. Reconocimiento de la subrepresentación de las mujeres en el nivel directivo, autorregulándolo para avanzar en una mayor igualdad de género.

- Cumplir o explicar, mediante un código de normas éticas que incluye la diversidad de género. Cuando no se cumple con dicha norma, la organización rinde cuentas públicamente sobre las razones por las cuales no se logra alcanzar igualdad entre hombres y mujeres en los niveles directivos.

- Cuotas de género. Obligación legal de garantizar igualdad de género en los cargos directivos de la organización. 
Según el estudio de la Dirección Nacional del Servicio Civil (2017), las mujeres tienen una representación menor respecto de la de los hombres en el sector público chileno, del $43 \%$ en cargos en el escalafón directivo y del 30\% en cargos directivos sometidos al SADP, lo que según Espinoza (2017) casi duplica la cantidad de mujeres que se desempeñan en puestos directivos en el sector privado y es similar a las cifras de administraciones públicas como la española, que alcanza un $32 \%$ de mujeres en cargos directivos (Carrancio, 2018).

Así, entre el 2004 y el 2017, se realizaron 3549 convocatorias de concursos en el SADP chileno (Tabla 6), y se dio lugar a una brecha de género que implica "la diferencia entre el porcentaje de mujeres nombradas y el porcentaje de postulaciones de mujeres" (Dirección Nacional del Servicio Civil, 2017, p. 19).

Tabla 6. Brecha de género en concursos del SADP por ministerio (2004-2017)

\begin{tabular}{cccc} 
Ministerio & $\begin{array}{c}\text { Tasa }(\%) \\
\text { postulación } \\
\text { Mujeres }\end{array}$ & $\begin{array}{c}\text { Tasa }(\%) \\
\text { selección } \\
\text { Mujeres }\end{array}$ & $\begin{array}{c}\text { Brecha de } \\
\text { género }\end{array}$ \\
\hline Agricultura & 19.6 & 16.0 & $-3.6 \%$ \\
Defensa Nacional & 17.1 & 25.0 & $7.9 \%$ \\
Deporte & 16.2 & 7.0 & $-9.2 \%$ \\
Desarrollo Social & 29.6 & 48.3 & $18.7 \%$ \\
Economía, Fomento y Turismo & 21.8 & 38.3 & $16.5 \%$ \\
Educación & 31.9 & 50.9 & $19.0 \%$ \\
Energía & 13.7 & 6.3 & $-7.4 \%$ \\
Hacienda & 17.3 & 22.6 & $5.4 \%$ \\
Interior y Seguridad Pública & 24.7 & 40.9 & $16.2 \%$ \\
Justicia & 20.2 & 28.3 & $8.1 \%$ \\
Medio Ambiente & 23.3 & 22.9 & $-0.3 \%$ \\
Minería & 16.3 & 8.3 & $-8.0 \%$ \\
Obras Públicas & 16.7 & 24.6 & $7.9 \%$ \\
Relaciones Exteriores & 21.6 & 36.4 & $-14.8 \%$ \\
Salud & 21.1 & 30.0 & $8.9 \%$ \\
\hline
\end{tabular}

\section{0}


Alta dirección pública y techo de cristal. Acceso de las mujeres a los puestos directivos en Chile

\begin{tabular}{cccc} 
Ministerio & $\begin{array}{c}\text { Tasa (\%) } \\
\text { postulación } \\
\text { Mujeres }\end{array}$ & $\begin{array}{c}\text { Tasa }(\%) \\
\text { selección } \\
\text { Mujeres }\end{array}$ & $\begin{array}{c}\text { Brecha de } \\
\text { género }\end{array}$ \\
\hline Trabajo y Previsión Social & 24.0 & 30.2 & $6.1 \%$ \\
Transportes y & 14.8 & 0.0 & -14.8 \\
Telecomunicaciones & 16.5 & 18.2 & $1.6 \%$ \\
Vivienda y Urbanismo & & & \\
\hline
\end{tabular}

Fuente: elaboración propia con base en Dirección Nacional del Servicio Civil (2017, pp. 20-21).

Según la Dirección Nacional del Servicio Civil (2017, p. 22 ), en el periodo bajo estudio, el promedio de la tasa de postulación de mujeres alcanzó el $22 \%$ sobre el total de 244116 personas que participaron en los concursos de ADP, esto en alguno de los dieciocho ministerios incluidos en la Tabla 6. En cambio, el promedio de la tasa de selección de mujeres alcanzó un 29.8\% sobre el total de 2111 personas seleccionadas en algún cargo de ADP.

Asimismo, la Dirección Nacional del Servicio Civil (2017) señala que en el 2005 se registró el porcentaje más alto de postulación de mujeres a los concursos de ADP entre los años del periodo analizado, un $27.1 \%$, mientras que en 2016 se registró el menor porcentaje de postulación de mujeres, con un $17.4 \%$ del total. En cuanto al porcentaje de selección, el más alto se registró en 2015, 37.6\%, y el más bajo en 2005, con un $24.6 \%$.

También respecto de la Tabla 6 , se observa que existen siete ministerios (Agricultura, Deporte, Energía, Medio Ambiente, Minería, Relaciones Exteriores y Transportes y Telecomunicaciones) con una brecha de género negativa. Se destaca el caso del Ministerio de Transportes y Telecomunicaciones, que registra la brecha más alta y es el único que nunca ha nombrado a una mujer en un cargo de ADP, según la Dirección Nacional del Servicio Civil (2017).

Asimismo, la Tabla 6 permite observar que el Ministerio de Educación y el Ministerio de Desarrollo Social registran

Sociedad No.77 
los mayores porcentajes de mujeres tanto en la tasa de postulación como en la tasa de selección, lo que confirma lo establecido por otros estudios en cuanto a la existencia de una segregación de tipo horizontal que concentra a las mujeres mayoritariamente en las áreas sociales del sector público chileno (Nasser, 2018; Moldovan, 2016; Cárdenas, Correa, y Prado, 2014; Sabharwal, 2013).

Tabla 7. Tasa de postulación y selección de mujeres por año en concursos ADP de primer y segundo nivel

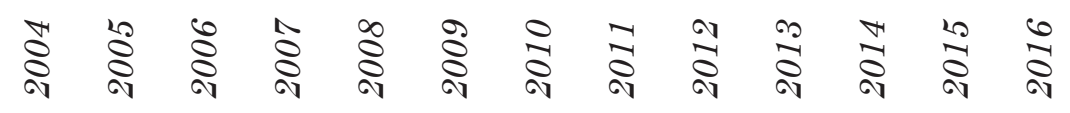

\begin{tabular}{|c|c|c|c|c|c|c|c|c|c|c|c|c|c|}
\hline 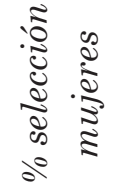 & 29 & 25 & 32 & 33 & 31 & 35 & 27 & 30 & 26 & 25 & 26 & 38 & 28 \\
\hline 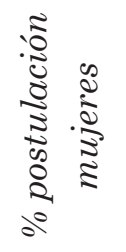 & 22 & 27 & 24 & 22 & 21 & 21 & 20 & 22 & 24 & 23 & 23 & 20 & 17 \\
\hline Brecha & 7 & -3 & 8 & 11 & 10 & 14 & 7 & 7 & 1 & 2 & 3 & 17 & 10 \\
\hline
\end{tabular}

Fuente: Dirección Nacional del Servicio Civil (2017, p. 23).

Finalmente, la Tabla 7 expone la distribución de la tasa de postulación y selección de mujeres a los concursos de primero y segundo nivel jerárquico del SADP según la Dirección Nacional del Servicio Civil (2017). Se observa que las mayores brechas se registraron entre los años 2007 y 2009, coincidentes con el primer mandato de la presidenta Michelle Bachelet y posteriores a la publicación del CBPL, ya mencionado. Asimismo, se destaca el periodo comprendido entre 2015 y 2016 , con altas brechas entre sus tasas, y que es coincidente con el segundo periodo de la presidenta Bachelet. 


\section{Metodología}

El estudio realizado examinó la problemática del TC en el sector público chileno desde una perspectiva cuantitativa, como ya se dijo, relacionada con el acceso de las mujeres a los cargos directivos incorporados al SADP, esto para responder a la pregunta de investigación: ¿existe una representación desequilibrada entre hombres y mujeres en los concursos de ADP en Chile? El periodo de análisis elegido fue 2010-2017, como también ya se mencionaba, que incluye el primer Gobierno del presidente Sebastián Piñera Echeñique y el segundo de la presidenta Michelle Bachelet Jeria.

Así, mediante una perspectiva deductiva, y a través de procedimientos de estadística descriptiva apoyada en la utilización de tablas y gráficos, se revisaron los resultados de los concursos de ADP para los cargos de primer y segundo nivel jerárquico en el periodo marcado. Se examinó una base de datos cuantitativos obtenidos desde el Servicio Civil a través de una petición de acceso a la información regida por la Ley de Transparencia. Se tomaron en cuenta los datos relacionados con la variable de género, y se contrastó esta con su distribución por año, nivel jerárquico, territorio y ministerio de concurso.

Finalmente, se compararon los resultados obtenidos en el presente estudio con los datos contenidos en el anuario de estadísticas de recursos humanos del sector público 20082017, elaborado por la DIPREs del Ministerio de Hacienda, esto último con el propósito de contrastar los datos con la distribución de los funcionarios públicos adscritos a los ministerios del Gobierno central de Chile.

\section{Resultados}

En la Tabla 8, se observa que entre 2010 y 2017 se desarrollaron un total de 2003 concursos públicos sometidos al

Sociedad No.77 
SADP chileno, y se observa que la mayor frecuencia se registró en el 2015, con cuatrocientos veintisiete concursos (21.3\%).

Tabla 8. Concursos de ADP realizados en Chile en el periodo 2010-2017

\begin{tabular}{cccc} 
Año & Frecuencia & Porcentaje & Porcentaje acumulado \\
\hline 2010 & 162 & 8.1 & 8.1 \\
2011 & 324 & 16.2 & 24.3 \\
2012 & 270 & 13.5 & 37.7 \\
2013 & 240 & 12.0 & 49.7 \\
2014 & 156 & 7.8 & 57.5 \\
2015 & 427 & 21.3 & 78.8 \\
2016 & 215 & 10.7 & 89.6 \\
2017 & 209 & 10.4 & 100.0 \\
Total & 2003 & 100.0 & \\
\hline
\end{tabular}

Fuente: elaboración propia.

Es importante recalcar que el SADP chileno sólo tiene quince años de funcionamiento (Espinoza, 2017; Llano, 2014), por lo que es factible asociar el aumento del número de concursos de ADP registrado en 2015 como una reacción a la crisis experimentada por el SADP durante los cambios de Gobierno de los años 2010 y 2014, que se tradujo en una serie de ajustes al sistema (Williamson e Irarrázabal, 2016) que aumentaron el número de cargos directivos de nivel primero y segundo sometidos a concursos de ADP, y que buscaban equilibrar nuevamente la discrecionalidad política con la meritocracia relacionada con las competencias técnicas para cada puesto directivo, como ya se había mencionado.

En la Figura 2, se presenta la distribución de los concursos de ADP para el periodo 2010-2017 entre servicios públicos adscritos y no adscritos. Estos últimos son aquellos que utilizan el SADP como mecanismo de reclutamiento y selección de los cargos directivos sin estar obligados por ley a hacerlo, 
y en los que los cargos nombrados a través de este sistema no están ligados a las condiciones de desempeño del SADP.

Figura 2. Distribución de concursos ADP

en Chile según servicios adscritos y no adscritos

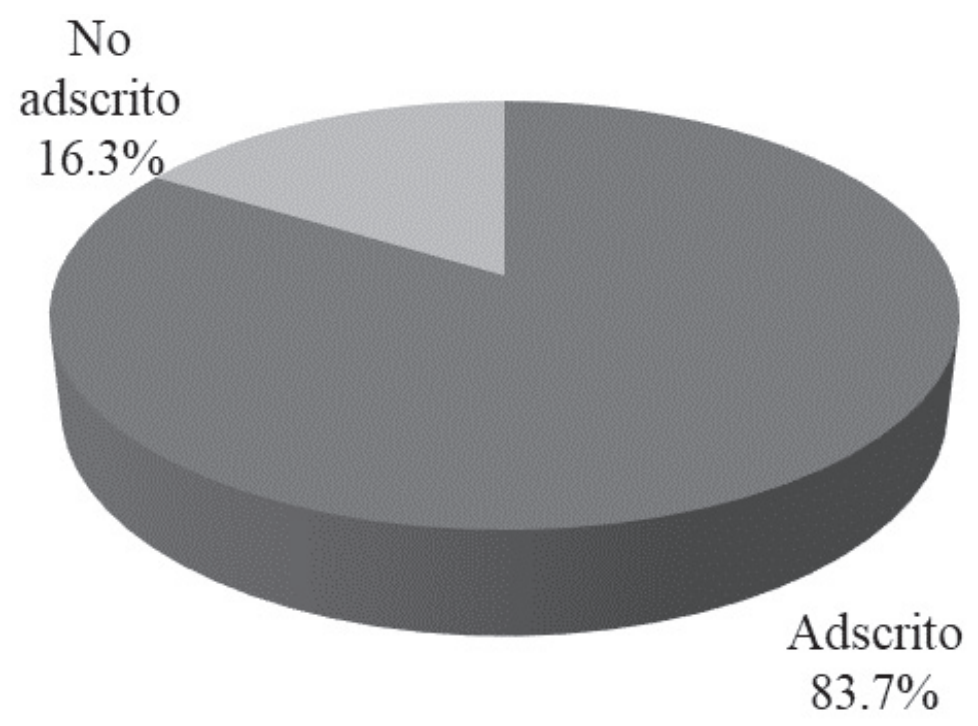

Fuente: elaboración propia.

Según la Figura 2, 1677 concursos de ADP (84\%) corresponden a servicios públicos adscritos al SADP, mientras que sólo trescientos veintisiete (16\%) pertenecen a instituciones no adscritas al sistema, lo que evidencia el alto grado de obligatoriedad que ha logrado el sADP en Chile, con una amplia cobertura de instituciones que están obligadas por ley a someter sus cargos directivos vacantes a dicho sistema.

La Figura 3, por su parte, presenta la distribución de los cargos concursados entre 2010 y 2017 según su nivel jerárquico y de acuerdo a lo establecido por el SADP chileno, lo que evidencia que los cargos directivos de primer nivel (nivel I) fueron trescientos sesenta y cuatro (18\%) durante el periodo analizado, mientras que los cargos de segundo nivel (nivel II) fueron 1639 (82\%). 
Figura 3. Distribución de cargos concursados en el SADP (2010-2017) según nivel jerárquico

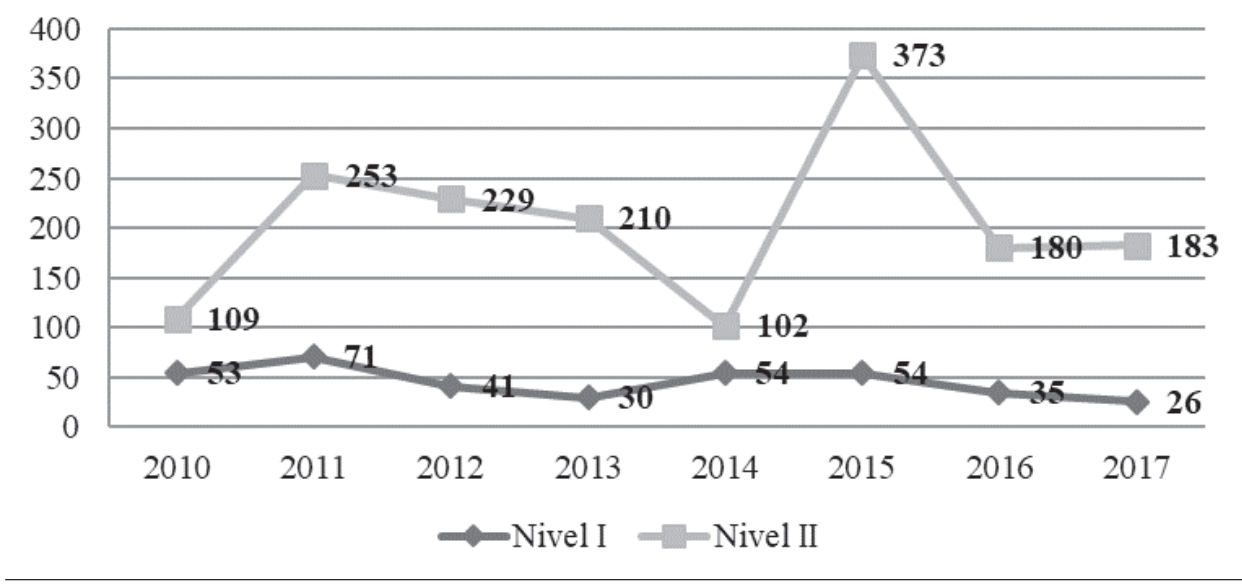

Fuente: elaboración propia.

Otra apreciación que surge de la Figura 3 es que en el segundo año de cada periodo de Gobierno, es decir, en 2011 y 2015, se produjeron importantes aumentos en el número de concursos de ADP, lo que es coincidente con lo planteado por Fraile (2018) en el sentido de que la mayor cantidad de concursos se relaciona con la aplicación de la facultad de los presidentes de la república de Chile de solicitar la renuncia a los directivos públicos. En ambos años, esto obedeció a la necesidad de los presidentes entrantes de contar con personas de confianza política en dichos puestos de alta dirección, lo que recuerda el sistema de botín ya aludido, mencionado por González et al. (2016).

En la Figura 4, se observa la distribución geográfica de los concursos de ADP realizados en Chile en el periodo bajo estudio. Las tres regiones más pobladas del país (Metropolitana, v y VIII) concentran la mayor cantidad de los 2003 concursos realizados. 
Figura 4. Distribución geográfica

de los concursos de ADP en Chile (2010-2017)

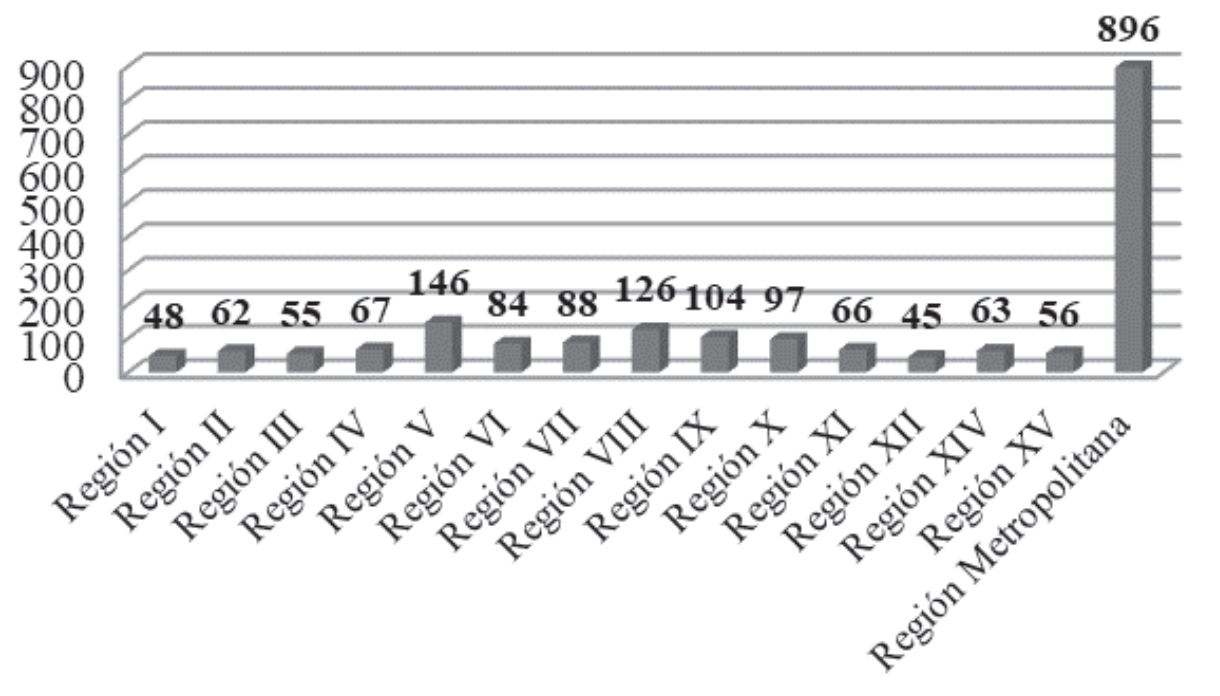

Fuente: elaboración propia.

También, es posible observar en la Figura 4 que las regiones más extremas al norte y sur del país (I y XII, respectivamente) registran la menor cantidad de concursos de ADP realizados, lo que en alguna medida responde tanto a las características históricas de Chile, que siempre ha tenido una fuerte concentración de las personas e instituciones en su zona central, como a la propia orientación del SADP, que concentra sus esfuerzos en las instituciones públicas pertenecientes al Gobierno central.

En cuanto a la distribución de los concursos chilenos de ADP según género, en la Figura 5 es posible observar que 1423 procesos (71\%) fueron adjudicados a hombres y quinientos ochenta cargos de ADP (29\%) a mujeres, lo que concuerda con los niveles de representación históricos del SADP chileno (Dirección Nacional del Servicio Civil, 2017) e implica resultados similares a los identificados en investigaciones sobre el TC en otros países (Carey y Dickinson, 2015; Carrancio, 2018; Marry y Pochic, 2017; Moldovan, 2016). 
Figura 5. Distribución por género de los concursos de ADP en Chile (2010-2017)

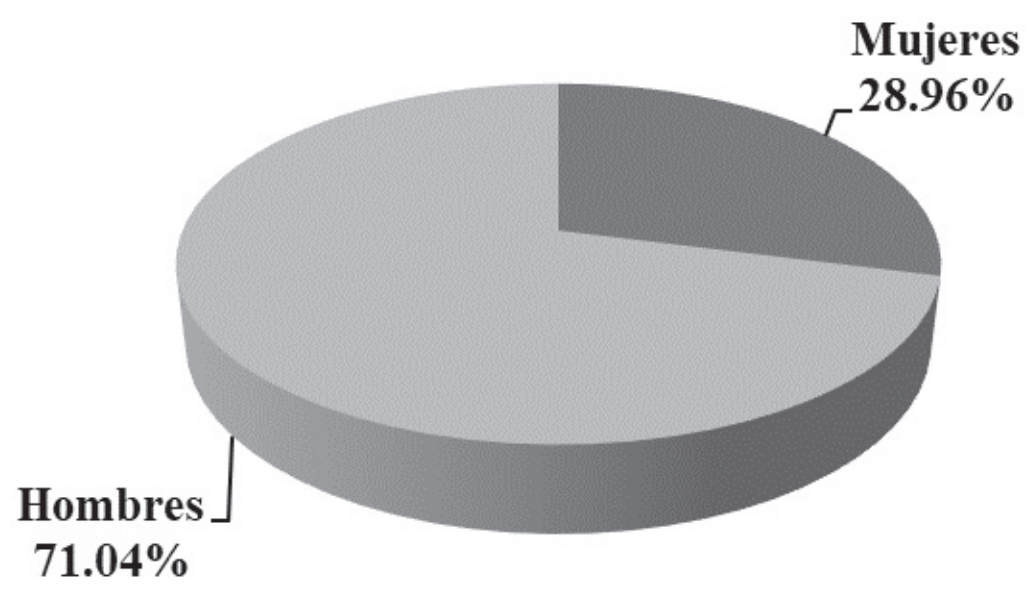

Fuente: elaboración propia.

Asimismo, la Figura 6 presenta el desglose de los resultados de los concursos de ADP por género por año, donde destaca que 2015 fue el año en que el mayor número de cargos directivos en el SADP fue adjudicado a mujeres, con ciento cuarenta y seis, lo que equivale al 34\% del total de cargos concursados ese año y supera el promedio de $29 \%$ del periodo bajo análisis. No obstante, también se evidencian niveles de adjudicación de puestos a mujeres por debajo de dicho promedio, como en el periodo entre 2011 y 2014, o en 2017, cuando se alcanzó un $26 \%$. 
Figura 6. Distribución por género y año

de los concursos de ADP en Chile (2010-2017)

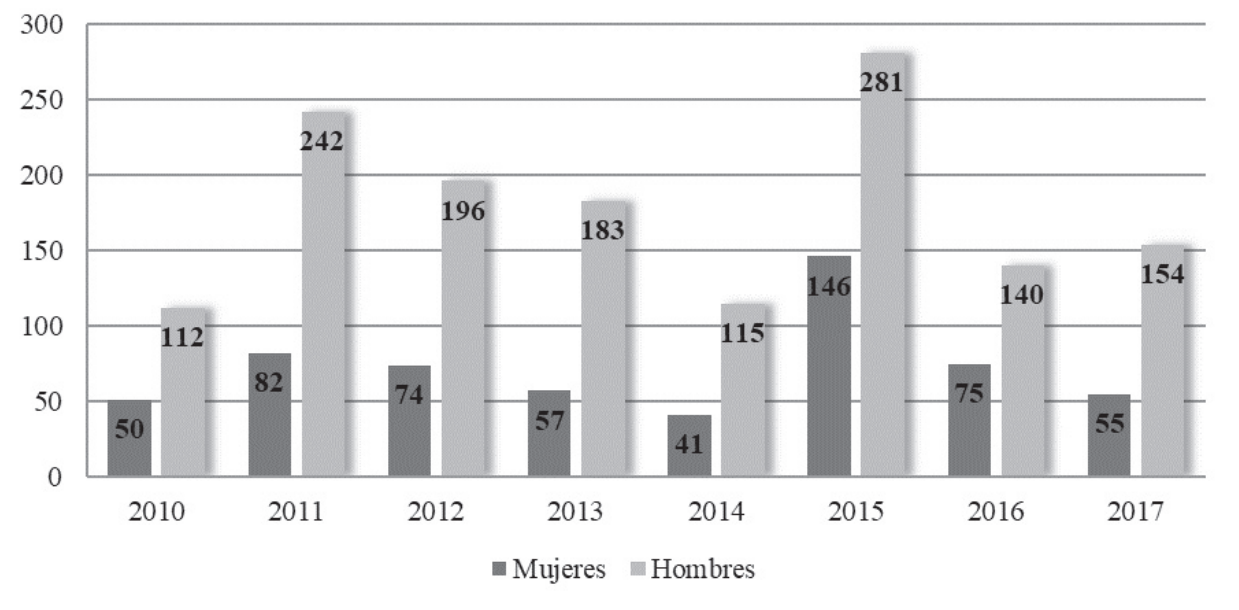

Fuente: elaboración propia.

Asimismo, si se analiza la variable de género respecto del nivel jerárquico de los cargos concursados en el SADP, se observa una mayor representación femenina en los cargos adjudicados en el nivel II, con un 30\% del total de ese segmento, mientras que en el caso de los cargos de nivel i el porcentaje alcanza un 24\% (Tabla 9).

Tabla 9. Distribución de concursos de ADP en

Chile por género y nivel jerárquico (2010-2017)

\begin{tabular}{cccc} 
Nivel & \multicolumn{2}{c}{ Género } & Total \\
& Mujeres & Hombres & 364 \\
Nivel I & 89 & 275 & 1639 \\
Nivel II & 491 & 1148 & 2003 \\
Total & 580 & 1423 &
\end{tabular}

Fuente: elaboración propia.

Lo expuesto en la Tabla 9 plantea al SADP chileno el desafío de aumentar la cantidad de mujeres que son seleccionadas en los concursos de ADP, especialmente para los cargos de primer nivel, que para el periodo analizado sólo alcanza- 
ron un $24 \%$ de ocupación femenina, por debajo de la meta del 30\% de participación de mujeres en cargos directivos comprometida para el Estado chileno en Beijing en 1995.

En la Tabla 10, se presenta la distribución de los cargos concursados en el SADP según región y género entre 2010 y 2017. Así, el mayor porcentaje de cargos adjudicados a mujeres a nivel regional se dio en la región XII, con el 44\%. Evidentemente, la mayor cantidad de mujeres contratadas a través del SADP se registró en la región Metropolitana, con doscientas cincuenta y nueve, pero estas equivalen sólo al $29 \%$ del total de cargos concursados en dicho territorio.

Tabla 10. Distribución de cargos de ADP en Chile según región y género (2010-2017)

\begin{tabular}{cccc}
\multicolumn{4}{c}{ Género } \\
Región & Mujeres & Hombres & Total \\
\hline Región I & 19 & 29 & 48 \\
Región II & 19 & 43 & 62 \\
Región III & 16 & 39 & 55 \\
Región IV & 23 & 44 & 67 \\
Región V & 44 & 102 & 146 \\
Región VI & 18 & 66 & 84 \\
Región VII & 26 & 62 & 88 \\
Región VIII & 32 & 94 & 126 \\
Región IX & 24 & 80 & 104 \\
Región X & 27 & 70 & 97 \\
Región XI & 18 & 48 & 66 \\
Región XII & 20 & 25 & 45 \\
Región XIV & 17 & 46 & 63 \\
Región XV & 18 & 38 & 56 \\
Región Metropolitana & 259 & 637 & 896 \\
Total & 580 & 1423 & 2003 \\
\hline
\end{tabular}

Fuente: elaboración propia.

En la Tabla 11, se presenta la distribución de los concursos de ADP realizados en los dos periodos de Gobierno que abar- 
Alta dirección pública y techo de cristal. Acceso de las mujeres a los puestos directivos en Chile

can los años del análisis, entre 2010 y 2013 para el caso del presidente Piñera, y 2014 y 2017 para el segundo periodo de Gobierno de la presidenta Bachelet.

Tabla 11. Distribución de cargos de concursos de ADP en Chile según ministerio y género por periodo presidencial (2010-2017)

\begin{tabular}{|c|c|c|c|c|c|c|c|}
\hline \multirow{2}{*}{ Ministerio } & \multicolumn{3}{|c|}{$2010-2013$} & \multicolumn{3}{|c|}{$2014-2017$} & \multirow{2}{*}{$\begin{array}{l}\% \text { var. } \\
\text { mujeres }\end{array}$} \\
\hline & $M$ & $H$ & Total & $M$ & $H$ & Total & \\
\hline Salud & 64 & 184 & 248 & 71 & 156 & 227 & $9.86 \%$ \\
\hline Energía & 2 & 10 & 12 & 1 & 13 & 14 & $-100 \%$ \\
\hline Agricultura & 7 & 45 & 52 & 11 & 42 & 53 & $36.36 \%$ \\
\hline $\begin{array}{c}\text { Trabajo y Previsión } \\
\text { Social }\end{array}$ & 24 & 60 & 84 & 30 & 46 & 76 & $20 \%$ \\
\hline Del Deporte & 1 & 16 & 17 & 2 & 26 & 28 & $50 \%$ \\
\hline Justicia & 9 & 30 & 39 & 17 & 29 & 46 & $47.06 \%$ \\
\hline $\begin{array}{c}\text { Interior y Seguridad } \\
\text { Pública }\end{array}$ & 14 & 24 & 38 & 15 & 13 & 28 & $6.67 \%$ \\
\hline Educación & 41 & 41 & 82 & 44 & 54 & 98 & $6.82 \%$ \\
\hline Hacienda & 10 & 51 & 61 & 11 & 44 & 55 & $9.09 \%$ \\
\hline Desarrollo Social & 14 & 16 & 30 & 14 & 10 & 24 & $0 \%$ \\
\hline Defensa Nacional & 4 & 13 & 17 & 2 & 11 & 13 & $-100 \%$ \\
\hline $\begin{array}{c}\text { Economía, Fomento y } \\
\text { Turismo }\end{array}$ & 30 & 47 & 77 & 33 & 57 & 90 & $9.09 \%$ \\
\hline Medio Ambiente & 7 & 22 & 29 & 6 & 20 & 26 & $-16.67 \%$ \\
\hline Relaciones Exteriores & 1 & 2 & 3 & 2 & 6 & 8 & $50 \%$ \\
\hline Obras Públicas & 10 & 26 & 36 & 9 & 25 & 34 & $-11.11 \%$ \\
\hline Minería & 1 & 17 & 18 & 1 & 9 & 10 & $0 \%$ \\
\hline $\begin{array}{c}\text { De la Mujer y la } \\
\text { Equidad de Género }\end{array}$ & 0 & 0 & 0 & 1 & 0 & 1 & $100 \%$ \\
\hline Vivienda y Urbanismo & 4 & 25 & 29 & 5 & 14 & 19 & $20 \%$ \\
\hline $\begin{array}{c}\text { Transportes y } \\
\text { Telecomunicaciones }\end{array}$ & 0 & 3 & 3 & 0 & 3 & 3 & $0 \%$ \\
\hline Total & 243 & 632 & 875 & 275 & 578 & 853 & \\
\hline
\end{tabular}

Fuente: elaboración propia. 
Según los resultados expuestos, cuatro ministerios (Energía, Defensa Nacional, Medio Ambiente y Obras Públicas) registraron una disminución de mujeres en cargos directivos de niveles I y II, mientras que en otros tres ministerios se produjo un estancamiento en la cantidad de mujeres (Desarrollo Social, Minería y Transportes y Telecomunicaciones). En cambio, en los Ministerios de la Mujer y Equidad de Género, Justicia y Agricultura se dieron los aumentos más importantes en el periodo bajo análisis.

Así, al considerar los resultados expuestos en las Tablas 9 y 11, es posible identificar tres ministerios en donde las mujeres se encuentran extremadamente subrepresentadas: Energía, Minería y Transportes y Telecomunicaciones. En este último, nunca ha sido nombrada una mujer mediante el SADP.

Finalmente, en la Tabla 12 se comparan los ministerios de Minería y Energía según género, nivel jerárquico y año. Entre dichas instancias, se ha nombrado en total únicamente a cinco mujeres en cargos de ADP en el periodo estudiado.

Tabla 12. Comparación entre Ministerio de Minería y Ministerio de Energía chilenos, según género, nivel y año de concursos de ADP

\begin{tabular}{|c|c|c|c|c|c|c|}
\hline & & & \multicolumn{4}{|c|}{ Ministerio } \\
\hline & & & \multicolumn{2}{|c|}{ Minería } & \multicolumn{2}{|c|}{ Energía } \\
\hline \multirow{2}{*}{ Género } & Muje & & \multicolumn{2}{|c|}{2} & \multicolumn{2}{|c|}{3} \\
\hline & Hom & & \multicolumn{2}{|c|}{26} & \multicolumn{2}{|c|}{23} \\
\hline \multirow{4}{*}{ Nivel } & \multirow{2}{*}{ Nivel I } & M & 0 & \multirow{2}{*}{14} & 0 & \multirow{2}{*}{5} \\
\hline & & $\mathrm{H}$ & 14 & & 5 & \\
\hline & \multirow{2}{*}{ Nivel II } & M & 2 & \multirow{2}{*}{14} & 3 & \multirow{2}{*}{21} \\
\hline & & $\mathrm{H}$ & 12 & & 18 & \\
\hline \multirow{4}{*}{ Año } & \multirow{2}{*}{2010} & M & 0 & \multirow{2}{*}{5} & 1 & \multirow{2}{*}{4} \\
\hline & & $\mathrm{H}$ & 5 & & 3 & \\
\hline & \multirow{2}{*}{2011} & M & 0 & \multirow{2}{*}{9} & 0 & \multirow{2}{*}{2} \\
\hline & & $\mathrm{H}$ & 9 & & 2 & \\
\hline
\end{tabular}

\section{2}


Alta dirección pública y techo de cristal. Acceso de las mujeres a los puestos directivos en Chile

\begin{tabular}{|c|c|c|c|c|c|c|}
\hline & & & \multicolumn{4}{|c|}{ Ministerio } \\
\hline & & & \multicolumn{2}{|c|}{ Minería } & \multicolumn{2}{|c|}{ Energía } \\
\hline \multirow{12}{*}{ Año } & \multirow{2}{*}{2012} & $\mathrm{M}$ & 1 & 1 & 1 & 4 \\
\hline & & $\mathrm{H}$ & 0 & 1 & 3 & 4 \\
\hline & \multirow{2}{*}{2013} & $\mathrm{M}$ & 0 & \multirow{2}{*}{3} & 0 & \multirow{2}{*}{2} \\
\hline & & $\mathrm{H}$ & 3 & & 2 & \\
\hline & \multirow{2}{*}{2014} & M & 0 & \multirow{2}{*}{2} & 0 & \multirow{2}{*}{1} \\
\hline & & $\mathrm{H}$ & 2 & & 1 & \\
\hline & \multirow{2}{*}{2015} & $\mathrm{M}$ & 1 & \multirow{2}{*}{6} & 0 & \multirow{2}{*}{1} \\
\hline & & $\mathrm{H}$ & 5 & & 1 & \\
\hline & \multirow{2}{*}{2016} & M & 0 & \multirow{2}{*}{0} & 1 & \multirow{2}{*}{5} \\
\hline & & $\mathrm{H}$ & 0 & & 4 & \\
\hline & \multirow{2}{*}{2017} & M & 0 & \multirow{2}{*}{2} & 0 & \multirow{2}{*}{7} \\
\hline & & $\mathrm{H}$ & 2 & & 7 & \\
\hline
\end{tabular}

Fuente: elaboración propia.

Respecto de la Tabla 12, es importante señalar que además de tratarse de ministerios que han nombrado muy pocas mujeres en cargos de ADP, ambas instituciones son fuertemente centralizadas, pues en el caso del Ministerio de Minería el 100\% de los cargos de ADP se ubica en la región Metropolitana, mientras que en el caso del Ministerio de Energía ese porcentaje alcanza un $74 \%$.

\section{Conclusiones}

El SADP chileno en sus primeros quince años de funcionamiento ha consolidado la mayoría de sus pilares fundamentales, y otorgado una especial atención a la limitación de la discrecionalidad de la autoridad en la designación de los cargos directivos de primer y segundo nivel jerárquico a personas por confianza política y no por mérito.

Sin embargo, en el contexto descrito en este trabajo, la participación de las mujeres en los cargos de ADP en Chile requiere de un fortalecimiento importante para proyectar dicho sistema en los próximos años, debido a que aún no 
se alcanza la meta propuesta en Beijing en 1995, especialmente respecto de los cargos directivos de primer nivel, en que la selección de mujeres promedia aún un $24 \%$.

Los resultados obtenidos confirman el planteamiento de la investigación, relacionado con la existencia de una representación desequilibrada entre hombres y mujeres en la adjudicación de los concursos de ADP en Chile para el periodo 2010-2017, que se expresa en que sólo el 29\% de los puestos directivos fue otorgado a una mujer, lo que configura a su vez un TC en el SADP chileno como expresión de una segregación vertical respecto de los hombres, específicamente en los cargos de primer nivel jerárquico de las instituciones públicas del Gobierno central. Esta situación se replica de una manera similar para los cargos de exclusiva confianza en los casos de ministros y subsecretarios, con sólo el $29 \%$ de los ministerios liderado por una mujer.

Por otro lado, se han identificado algunos antecedentes adicionales para describir la existencia de una segregación horizontal a nivel ministerial, relacionada con el concepto de muros de cristal: determinados sectores del Gobierno central chileno están más feminizados, como los ministerios de Salud, Educación, Economía y Fomento y Turismo, que muestran una mayor presencia de mujeres en detrimento de otros ámbitos ministeriales en que no se ha seleccionado a una mujer para un cargo de ADP en el periodo estudiado, como los ministerios de Minería, Energía y muy especialmente el de Transportes y Telecomunicaciones.

También, la discrecionalidad de las autoridades responsables de nombrar a los altos directivos públicos chilenos ha estado presente tanto en la génesis como en la implementación del SADP chileno, y ha privilegiado en un importante porcentaje de los casos la confianza política sobre las competencias técnicas. Esto ha impulsado el clientelismo político, asociado al establecimiento de un posible sistema de botín en el nivel directivo de la administración pública 
chilena (González et al., 2016) que ha favorecido mayoritariamente a los hombres, sin una acción afirmativa que dirija dicho favoritismo hacia las mujeres.

A partir del diagnóstico realizado, y desde la literatura expuesta en este artículo, se identifican estrategias que podrían considerarse para mejorar la representación equitativa entre hombres y mujeres en cargos directivos concursados en el SADP chileno. Se proponen tres en específico:

1. En igualdad de condiciones en los concursos de ADP, se deben aplicar acciones afirmativas en favor de las mujeres (Servicio Nacional de la Mujer, 2008), especialmente para los cargos de primer nivel jerárquico;

2. Las instituciones públicas adscritas al SADP deben rendir cuenta a la ciudadanía respecto del cumplimiento del CBPL o explicar las razones por las cuales no se avanza en la representación equitativa de hombres y mujeres en el nivel directivo de cada institución pública (Comunidad Mujer, 2015); y

3. Eliminar las culturas organizativas discriminatorias y poco comprensivas (Programa de las Naciones Unidas para el Desarrollo, 2014), especialmente en lo relativo a la conciliación trabajo-familia o la baja sororidad como principales barreras que generan el TC (Gaete, 2018a y 2018b; Gaete, Álvarez, y Ramírez, 2019).

Finalmente, los resultados del presente estudio permiten proyectar otras investigaciones con diseños cuantitativos o cualitativos que indaguen específicamente en la identificación y descripción de cuáles son las principales barreras u obstáculos que deben enfrentar las mujeres para acceder a los puestos de alta dirección pública en Chile, especialmente a través de una perspectiva cualitativa (Gaete, 2018a y 2018b; Gaete, Álvarez, y Ramírez, 2019).

Asimismo, otra línea de investigación importante se relacionaría con el estudio de los aspectos socioculturales 
presentes en los procedimientos y criterios de selección que se aplican en los concursos de ADP en Chile y que facilitan la reproducción de las desigualdades hacia las mujeres, de manera similar al estudio desarrollado por la Corte Suprema de Justicia de Argentina (2014), de modo que se puedan analizar las bases de los llamados a concurso público y el desarrollo de las diferentes etapas del proceso de selección de ADP en los que postulan las mujeres, especialmente respecto de los criterios utilizados para evaluar las competencias laborales de los postulantes a los cargos directivos.

Bibliografía

Caceres-Rodriguez, R. (20I3). The glass ceiling revisited. Moving beyond discrimination in the study of gender in public organizations. Administrations \& society, 45(6), 674709. doi https://doi.org//0.1 I77/00953997| | 429104

Cárdenas, A., Correa, N., y Prado, X. (20I4). Segregación laboral y género: tendencias y desafíos relativos al mercado laboral de la salud y la educación en Chile. Revista Polis, 38, I-I9. doi http://dx.doi.org/I0.4067/ S07I8-656820I40002000I8

Carey, G., y Dickinson, H. (20 I5). Gender in Public Administration: Looking Back and Moving Forward. Australian Journal of Public Administration, 74(4), 509-5 I 5. doi http:// doi.org/I0.1 I I I/I467-8500.12172

Carrancio, C. (20I8). El techo de cristal en el sector público: acceso y promoción de las mujeres a los puestos de responsabilidad. Revista española de sociología, 27(3), 475-489. doi https://doi.org// 0.22325/fes/res.2018.17

Carrera, C. (2016). La discriminación por razones de género en la administración pública: un tema pendiente [ponencia presentada en el XXI Congreso internacional del CLAD sobre la reforma del Estado y de la administración pública]. Centro Latinoamericano de Administración para el Desarrollo: Santiago de Chile. Recuperado de http:// www2.congreso.gob.pe/sicr/cendocbib/con4_uibd.

\section{6}


nsf/ I 254E46E28252C98052580C8005 I AF0A/\$FILE/ Bibliografía carrecar.pdf

Comisión Económica para América Latina y el Caribe (2014). Informe nacional Chile. División de Asuntos de Género. Recuperado de https:/www.cepal.org/mujer/noticias/ paginas/3/5I823/Informe_Chile_Beijing_20.pdf

Comisión Nacional de Derechos Humanos de México (2017). Estudio sobre la igualdad entre mujeres y hombres en materia de puestos y salarios en la administración pública federal (AfP). Programa de Asuntos de la Mujer y de Igualdad entre Mujeres y Hombres. Recuperado de http:// www.cndh.org.mx/sites/all/doc/Informes/Especiales/ Estudio-igualdad-20180206.pdf

Comité para la Eliminación de la Discriminación contra la Mujer (1979). Convención sobre la eliminación de todas las formas de discriminación contra la mujer. Recuperado de https://www.ohchr.org/Documents/Professionallnterest/cedaw_SP.pdf

Comunidad Mujer (2015). Mujer y trabajo: la necesaria incorporación de las mujeres a la alta dirección. Recuperado de http://dev.comunidadmujer.cl/biblioteca-publicaciones/wp-content/uploads/20 I 5/03/BOLETIN-MARZO-20I5-VF.pdf

Corte Suprema de Justicia de Argentina (20I4). Acceso de las mujeres a la Magistratura:perfil de los/las postulantes a los concursos. Oficina de la Mujer. Recuperado de https:// www.csjn.gov.ar/om/docs/techo.pdf

Cuadrado, I., y Morales, J. (2007). Algunas claves sobre el techo de cristal en las organizaciones. Revista de psicología del trabajo y de las organizaciones, 23(2), 183-202. Recuperado de https://journals.copmadrid.org/jwop/ art/6395ebd0f4b478I45ecfbaf939454fa4

Dirección de Presupuestos (2018). Estadísticas de recursos humanos del sector público 2008-2017. Recuperado de 
http://www.dipres.gob.cl/598/articles-1 76466_doc_pdf. pdf

Dirección de Presupuestos (2019). Informe trimestral de los recursos humanos del sector público. Recuperado de http:// www.dipres.gob.cl/598/articles- I89247_version_PDF.pdf

Dirección Nacional del Servicio Civil (2017). Condiciones y representación de las mujeres en el sector público. Santiago de Chile: Unidad de Estudios y Cooperación. Recuperado de https://documentos.serviciocivil.cl/ actas/dnsc/documentService/downloadWs?uuid=7af47d89-d5dc-426c-8487-c8। 4abd9bd4c

Egaña, R. (2015). Experiencia de la alta dirección pública chilena a doce años de su implementación [ponencia presentada en el $x x$ Congreso internacional del CLAD sobre la reforma del Estado y de la administración pública]. Centro Latinoamericano de Administración para el Desarrollo: Lima. Recuperado de https://documentos.serviciocivil. $\mathrm{cl} /$ actas/dnsc/documentService/downloadWs?uui$d=379$ acf39-43c8-4f23-b458-0ac60b4cebd9

Espinoza,V.(20I7).Sistema deAlta Dirección Pública chileno: evolución de un sistema que se consolida como referente de acceso meritocrático a la dirección pública [ponencia presentada en el XXII Congreso internacional del CLAD sobre la reforma del Estado y de la administración pública]. Centro Latinoamericano de Administración para el Desarrollo:Madrid. Recuperado de https://documentos. serviciocivil.cl/actas/dnsc/documentService/downloadWs?uuid=cee644e8-c6ae-4c3a-8fa I-cd044b2f0852

Fraile,A. (2018). Trece años de funcionamiento del Sistema de Alta Dirección Pública en Chile. Un modelo de gestión incompleto. En I.Aninat, y S. Razmilic (Eds.), Un Estado para la ciudadanía. Estudios para su modernización (pp. 36I-402). Santiago de Chile: Centro de Estudios Públicos.

\section{8}


Gaete, R. (20/8a).Acceso de las mujeres a los cargos directivos: universidades con techo de cristal. Revista cs, 24 , 67-90. doi https://doi.org//0.18046/recs.i24.243 I

Gaete, R. (20I8b). Conciliación trabajo-familia y responsabilidad social universitaria: experiencias de mujeres en cargos directivos en universidades chilenas. Revista digital de investigación en docencia universitaria (RIDU), I 2(I), 8I-I00. doi https://doi.org/I0.19083/ridu.12.6I5

Gaete, R., Álvarez, J., y Ramírez, M. (2019). Reflexiones y experiencias de profesoras-investigadoras mexicanas sobre el techo de cristal. Revista Calidad en la educación, 50, 457-49I. doi http://dx.doi.org//0.316/9/caledu. $\mathrm{n} 50.494$

González, B., Olivares, A., Abarca, P., y Molina, E. (2016). Servicio Civil en Chile, análisis de los directivos de primer nivel jerárquico (2003- I3). Revista Administração Pública, 50(I),59-79. doi http://dx.doi.org/ / 0.1590/00347612145767

Gutiérrez, A. (2016). La institucionalización del enfoque de género en Chile 1990-20 I5. En I. Cienfuegos, y F.Penaglia (Eds.), Manual de administración pública (Pp. 355-38I). Santiago de Chile: RiL Editores.

lacoviello, M., Llano, M., y Ramos, C. (2017). Alta dirección pública latinoamericana: marchas y contramarchas. Revista de gestión pública, 6(2), I73-2।4. Recuperado de http://www.revistadegestionpublica.cl/Vol_VI_No_2/ lacovielloLlanoRamos.pdf

Instituto de Investigación de las Naciones Unidas para el Desarrollo Social (2006). Igualdad de género. La lucha por la justicia en un mundo desigual. Francia: Naciones Unidas. Recuperado de http://www.unrisd.org/80256B3C005BC CF9/httpNetlTFramePDF?ReadForm\&parentunid=54B9DD585F89 IF6DCI 257 I 630047FFF0 \& parentdoctype=book\&netitpath=80256B3C005BCCF9/(httpAux- 
Llano, M. (20I4). Diagnóstico institucional del servicio civil en América Latina:Chile. Nota técnica IDB-TN-678. Recuperado de https://publications.iadb.org/bitstream/handle /I I 3 1 9/6622/ICS\%20TN\%20Diagn\%C3\%B3stico\%20 institucional\%20\%20del\%20servicio\%20civil\%20en\%20 \%20Am\%C3\%A9rica\%20Latina-Chile.pdf?sequence= I Longo, F. (2013). Diez años de alta dirección pública en Chile [ponencia presentada en el XVIII Congreso internacional del CLAD sobre la reforma del Estado y de la administración pública]. Centro Latinoamericano de Administración para el Desarrollo: Montevideo. Recuperado de https:// cladista.clad.org/bitstream/handle/ I 23456789/7/ 90/007 4457.pdf?sequence $=$ I \&isAllowed $=y$

Macarie, F., y Moldovan, O. (20|4). The Glass Ceiling in the Public Sector: A case Study Regarding Romanian Elected and Appointed Officials. Managerial Challenges of the Contemporary Society, 7(2), I0-16. Recuperado de https:// papers.ssrn.com/sol3/papers.cfm?abstract_id $=2642745$ Maillet, A. (2015). Fortalecimiento del Sistema de Alta Dirección Pública en Chile: una mirada comparada. Chile: Servicio Civil. Recuperado de https://www.serviciocivil.cl/ wp-content/uploads/2018/03/5.-Fortalecimiento-del-sADP-en-Chil.-Una-mirada-comparada.pdf

Marry, C., y Pochic, S. (2017). Le plafond de verre en France: Le public plus égalitaire que le privé? Cadernos de pesquisa, 47(163), I48-166. doi http://dx.doi. org/I0.1590//98053/44299

Moldovan, O. (2016). Representative Burocracy in Romania? Gender and Leadership in Central Public Administration. Transylvanian Review of Administrative Sciences, 48, 66-83. Recuperado de http://rtsa.ro/tras/index.php/tras/ article/view/484 
Nasser, S. (20I8). Boxed Women in Public Administration -Between Glass Ceilings and Glass Walls: A Study of Women's Participation in Public Administration in the Arab States. Journal of International of Women's Studies, 19(3), I52-I7|. Recuperado de https://vc.bridgew.edu/ cgi/viewcontent.cgi?referer=https://www.google.cl/\&httpsredir $=\mid$ \&article $=203 \mid$ \&context $=$ jiws

Organización Internacional del Trabajo (2017). Panorama mundial: la mujer en cargos directivos y de gestión en las organizaciones de empleadores, documento $n^{\circ}$ I 7. Ginebra: Oficina Internacional del Trabajo, Oficina de Actividades para los Empleadores (ACT/EMP). Recuperado de https:// www.ilo.org/public/spanish/dialogue/actemp/downloads/ publications/wibm_globalsnapshot_2017_sp.pdf

Pliscoff, C. (20I6). Alta dirección pública: elementos teóricos y reflexiones a partir del caso chileno. En I. Cienfuegos, y F. Penaglia (Eds.), Manual de administración pública (pp. 383-40 I). Santiago de Chile: RiL Editores.

Programa de las Naciones Unidas para el Desarrollo (20I4). lgualdad de género en la administración pública. Nueva York: PNUD. Recuperado de https://iknowpolitics.org/ sites/default/files/gepa_report_sp_web.pdf

Ortega, D. (2016). Análisis de los factores impulsores y obstaculizadores en el avance de la implementación del Sistema de Alta Dirección Pública (SADP) de Chile del 2003 al 20 I 4. Recuperado de http://www.mgpp.cl/wp-content/ uploads/2017/04/I36Denis-Ortega-2.pdf

Sabharwal, M.(20I3). From glass ceiling to glass cliff: Women in senior executive service. Journal of public administration research and theory, 25(2), 399-426. doi https://doi. org/ 10.1093/jopart/mut030

Servicio Civil (2018). Alta dirección pública. Camino a la profesionalización del Estado (2003-2017). Recuperado de https://documentos.serviciocivil.cl/actas/dnsc/docu- 

mentService/downloadWs?uuid=dd29398f-ec44-4e8c$8 \mathrm{bcc}-15 \mathrm{~d} 9 \mathrm{~d} 28 \mathrm{e} 5 \mathrm{~b} 0 \mathrm{a}$

Servicio Nacional de la Mujer (2008). Código de buenas prácticas laborales sobre no discriminación para la administración central del Estado. Santiago de Chile: Gobierno de Chile. Recuperado de http://www.comunidadmujer.cl/biblioteca-publicaciones/wp-content/uploads/20 I I/0 I/C\%C3\%B3digo-de-Buenas-Pr\%C3\%A I cticas-Laborales.pdf Williamson, C., e Irarrázabal, I. (20I6). Modernización del Sistema de Alta Dirección Pública: un paso necesario pero insuficiente. Apuntes legislativos, (3I). Recuperado de https://politicaspublicas.uc.cl/wp-content// uploads/2016/06/Modernizaci\%C3\%B3n-del-Sistema-de-ADP.pdf

Zuvanic, L. (2016). En busca del destino: hacia la profesionalización de la alta dirección pública en América Latina. Documento de políticas públicas, no. I80. Recuperado de https:// www.cippec.org/wp-content/uploads/2017/03/I000.pdf 